PPPL- -2894

DE93 $01 j 495$

\title{
The Iterative Monte Carlo Technique for Collisionless Plasma Flow to a Surface
}

\author{
C. S. Pitcher* \\ Princeton Plasma Physics Laboratory, Princeton University, Princeton, P.O. Box 451, NJ 08543, \\ USA \\ * Canadian Fusion Fuels Technology Project, Toronto, Canada. Present Address: Univer- \\ sity of Toronto, 4925 Dufferin Street, Downsview, Ontario, Canada, M3H 5T6.
}

\begin{abstract}
A new technique for modelling the boundary plasma of magnetic fusion devices is described. The technique represents a natural extension of existing Monte Carlo codes, which are presently constrained to have the plasma background specified by either measurements or predictions from plasma fluid codes. The new approach, the Iterative Monte Carlo (IMC) technique, self-consistently determines the ambipolar electric field in the plasma by feeding back into the simulation the evolving plasma density using the Boltzmann relation.

The IMC technique is applied, for demonstrative purposes, to the problem of collisionless one-dimensional plasma flow to a surface. Such a problem has previously been solved exactly using kinetic approaches in the published literature using two different particle source functions. Good agreement between the IMC results and the exact solutions is obtained.
\end{abstract}




\section{Introduction}

One of the most challenging obstacles facing magnetic thermonuclear fusion research is the plasma-surface interaction problem [1]; that is, the interaction of the plasma boundary with the internal surfaces of the fusion device. In this region, magnetic field lines intersect material boundaries, such as limiters or divertor plates, and thus these structures are subjected to high particle and heat fluxes from the plasma. The energetic fluxes can both sputter and evaporate the surface, releasing impurities which then contaminate the plasma. In addition, the helium "ash" that originates in the plasma core is neutralized in the boundary region and must be removed from the boundary by vacuum pumps [2]. The technological problems associated with this interaction are many, and consequently large research efforts exist world-wide to address them. Research is approximately split between boundary plasma modelling and boundary plasma measurements. This paper discusses a new method of modeiling the plasma boundary.

One useful form of boundary plasma modelling that has been developed over the last decade is impurity Monte Carlo codes [3,4]. In this type of modelling, no attempt is made at calculating the background boundary plasma conditions; instead, the background plasma conditions are assumed, using for example measurements from experiments or results from plasma fluid codes. The Monte Carlo codes primarily address the impurities in the boundary plasma, determining impurity production and transport, erosion/redeposition patterns and impurity radiation distributions. This entails "launching" individual impurity particles from the material surface and following them in a plasma grid assuming classical parallel field transport processes, i.e. diffusion, friction, temperature gradient forces, ambipolar electric fields, ion thermalization, etc. In addition, many of the known atomic processes can be included, i.e. ionization, line radiation, recombination, etc. The atom/ion is followed in the plasma grid until it returns to the surface, whence, another particle is launched. As an ion passes through a "bin" in the plasma grid, an amount of "density" is left in that bin in inverse proportion to the time that the ions resides in it. The trajectories, radiation densities and erosion/deposition patterns are summed within the spatial grid by the code for each particle until reasonably accurate distributions are established.

This type of modelling has been found to be most useful as a means for interpreting experimental results, for example, comparing predicted patterns for impurity and radiated power 
emission [5] or erosion/redeposition [6] when the background plasma has been measured. It does not suffer convergence problems but has no predictive capabilities for the background plasma.

This paper suggests a new way of performing similar Monte Carlo boundary plasma calculations, while removing the requirement that the background plasma be predetermined; that is, the new technique derives the background plasma conditions. The technique is basically an extension of the Monte Carlo (MC) technique outlined above, however, with one main difference. While in the standard MC technique, a steady, unchanging background plasma is assumed for every trajectory, in the present paper the plasma density distributions evolve as the simulation proceeds and are fed back into the simulation. With time, the distributions converge on the steady-state solution. We call this approach the "Iterative Monte Carlo" (IMC) technique.

\section{Collisionless One-Dimensional Plasma Flow to a Surface}

In this paper the IMC technique is demonstrated by applying it to a plasma problem with known analytic solutions. In the context of boundary modelling, the most straightforward comparison is with collisionless one-dimensional plasma flow to a surface. The geometry is shown in Fig. 1. A region with a volume source of proton and electron pairs with temperatures $T_{i}$ and $T_{e}$, respectively, is bounded on either side by perfectly absorbing plates (for charge) spaced a distance $2 \mathrm{~L}$ apart. This geometry approximates, in one dimension, the situation in the scrape-off layer of a magnetic fusion device, i.e. the plates represent either the limiter or divertor structures separated by a connection length of $2 \mathrm{~L}$ [7]. In this case the volume source in the one-dimensional problem represents the cross-field particle source due to diffusion from the confined plasma region into the scrape-off layer.

This type of problem has been dealt with extensively in the literature [8-12], going back to Tonks and Langmuir in 1929 [8]. However, for comparison in this paper we will compare the IMC technique with results from two more recent papers, Emmert et al in 1980 [9] and Scheuer and Emmert in 1988 [11]. In the case of Emmert et al, the plasma ions are assumed to be born with a source velocity distribution S (see Fig. 2) which would give rise to a Maxwellian ion velocity distribution function if there were no ambipolar electric field in the plasma. The spatial source of ions is uniform, they are assumed to be collisionless and the electrons are assumed to 
have a Maxwell-Boltzmann distribution. The last assumption thus implies, assuming plasma quasi-neutrality, that the ion and electron density $n$ at any point is given by the Boltzmann relation,

$$
n=n_{0} \exp \left[\frac{e \phi}{k T_{e}}\right]
$$

where $n_{0}$ is the density at the symmetry point $x=0, e$ is the electronic charge, $k$ is Boltzmann's constant and $\phi$ is the plasma space potential with respect to that at the symmetry point. Thus, the plasma density at any point gives the space potential from which the electric field can be determined.

Fig. 1 shows the qualitative trends of the plasma density and space potential, both are maximum at the symmetry point and decrease towards the plates. In the case of the density, the gradient towards the plates is a consequence of the sink action at the surface for charged particles. In the case of the potential, the curve shown in Fig. 1 gives rise to a potential well for the electrons (hence the applicability of the Boltzmann relation) and an accelerating ambipolar electric field for the ions.

\section{Iterative Monte Carlo Technique}

The plasma grid is illustrated in Fig. 3. Since the distributions are symmetric about $x=0$ we consider only the region $0<x<L$ and reflect particles at the $x=0$ boundary. In anticipation that the spatial variations of plasma density and potential will be greatest near the plate $(x=L)$, we specify a higher concentration of the bins in this region compared with the symmetry point. The total number of bins, $\mathrm{N}+1$, is typically in the range of 10 to 50 . Before beginning the simulation, the population of each bin is initialized with some small number, typically 100 , thus initially giving a flat density profile and therefore no electric field. Ions are then launched, one at a time, starting at random locations with the velocity distribution shown in Fig. 2 and are followed within the plasma grid until they are absorbed by the plate.

The ion motion between time-steps is governed by the following equation of motion,

$$
\Delta x=\frac{1}{2} \frac{e}{m} E \Delta t^{2}+v_{0} \Delta t
$$


where $m$ is the ion mass, $E$ is the electric field and $\nu_{0}$ is the initial velocity. The change in velocity is given by,

$$
\Delta v=\frac{e E}{m} \Delta t
$$

The time-step is varied in proportion to the bin size according to

$$
\begin{gathered}
\Delta t=\frac{c h}{c_{S}} \\
c_{S} \equiv\left[\frac{k\left(T_{i}+T_{e}\right)}{m}\right]^{1 / 2}
\end{gathered}
$$

where $\mathrm{h}$ is the distance between bin centres (see Fig. 3), $c_{s}$ is the ion acoustic speed and $\mathrm{c}$ is a constant for a given simulation, typically in the range of 0.01 to 0.1 . The variable time-step and bin size ensure that adequate time and spatial resolution is available in regions where the density and potential may vary rapidly in space. It also means that the increment in bin population at each step is always the same regardless of the magnitude of the time-step or bin size; the increment is taken as 1 .

The electric field at a given point is determined using the populations of the nearby bins and the Boltzmann relation,

$$
\begin{gathered}
E=-\frac{\Delta \phi}{h} \\
\frac{e \Delta \phi}{k T_{e}}=\ln \left[\frac{n_{i+1}}{n_{i}}\right]
\end{gathered}
$$

It is convenient to normalize the position $\mathrm{x}$ and the particle velocity $\mathrm{v}$ according to the following definitions,

$$
\begin{gathered}
s \equiv \frac{x}{L} \\
u \equiv v\left[\frac{2 k T_{i}}{m}\right]^{-1 / 2}
\end{gathered}
$$




\section{Evolution}

As the ion travels through the plasma grid it deposits "density" in the bins. This modifies the density distribution in the space, thus changing the potential distribution through the Boltzmann relation. This changes the electric field and thus feeds back on the ion motion through Eqns. 2 and 3. At the early stages of the simulation, the number of counts per bin is low and thus the density distribution has a high level of statistical noise. This causes the ion paths to take complicated and sometimes unrealistic trajectories through velocity phase space. For example, in the presence of noise it is possible for ions, born with low values of initial velocity, to exhibit oscillatory motion as they are reflected by peaks in the noisy density/potential distribution. Such an example is demonstrated in Fig. 4, which shows the density distribution just before and just after an ion with oscillatory motion. In this case, approximately $\sim 200$ particles had been run in the simulation with $T_{i} / T_{e}=0.1$. Also shown in the figure is the trajectory of the oscillating particle with the trajectory time-step number plotted on the right vertical axis. The ion is born within a potential well, created by the noise in the density distribution, in the vicinity of $s$ 0.915. In the presence of the initial density distribution, the ion has insufficient kinetic energy to escape the well and thus oscillates within.it. As time proceeds, the ion deposits "density" within the bins of the well, thus raising the cell population and decreasing the depth of the well. In time, the ion is able to escape and proceed through the plasma grid to deposit at the plate.

As mentioned, the oscillatory behaviour of the particle is not realistic, given that in this physical problem a particle should experience no more that one turning point. However, in approaching a realistic solution of the problem, the repeated oscillations serve to smooth over the distribution, i.e. they tend to fill in "valleys" in the density distribution, which are only present at the beginning of the simulation due to statistical noise. This is demonstrated by comparing the density distribution before and after the oscillating particle in Fig. 4. This type of behaviour becomes more pronounced at low values of $T_{i} / T_{e}$, where the ion motion, because of the lower ion velocities, is more easily affected by density noise.

In Fig. 5, two history traces are given for the evolution of the density at the plate as a function of particle number for the case $T_{i} / T_{e}=1$. The first shows results from the IMC technique, where the density distribution (and thus the electric field) is updated at every time-step. In the second, the feed-back between the density (i.e. the cell population) and the electric field is not included, instead, the exact electric field, derived from Emmert et al, is used throughout the 
entire simulation. The second case thus corresponds to the standard MC approach, where the background electric field is predetermined and held constant. Comparison of the two curves demonstrates the efficiency of the feed-back mechanism to derive the correct electric field. Also shown in Fig. 5 is the exact solution according to Emmert et al, $n(L) / n_{0}=0.667$. In both the IMC and MC cases, the density settles to within $\pm 10 \%$ of the correct value within 1000 particles, although the simulation which uses the exact electric field appears to settle somewhat faster.

\section{Detailed Comparison with Emmert et al}

In this section we make further comparisons with the results from Emmert et al [9] who have solved this problem analytically. In their work the solutions to the density and potential distributions represent the solution to a transcendental equation, which has been solved numerically for presentation here. The reader is referred to their paper for the exact mathematical expressions.

In Fig. 6 the exact density distributions as a function of $s \equiv x / L$ for $T_{i} / T_{e}=0.1,1.0,10.0$ are given from Emmert et al. This range of ion temperature is representative of the bounds typically observed in the boundary of tokamak plasmas [1]. Also shown, for comparison, are the corresponding results using the IMC technique. Reasonable agreement is obtained. It should be noted that, although some "noise" is present on the IMC results, any level of agreement can be obtained between the IMC results and the exact results depending on the bin size, the time-step and the number of particles.

As expected, in Fig. 6 the density decreases towards the plate due to the sink action for charged particles at the surface. The density gradient depends on the magnitude of the ion temperature compared with the electron temperature, the gradient being greater at low values of $T_{i} i T_{e}$ and smaller at high values of $T_{i} / T_{e}$. The large density gradient at low values of $T_{i} / T_{e}$ produces a strong electric field which accelerates the ions toward the plate, thus compensating for their lack of thermal velocity.

Fig. 7 shows the normalized potential next to the plate $-e \phi_{1} / k T_{e}$ as a function of the ratio of ion temperature to electron temperature $T_{i} / T_{e}$ for the exact solution by Emmert et al and for a number of cases using the IMC technique. The agreement is good, in general, but tends to be 
better at high ion temperature compared to lower ion temperatures. This, as mentioned earlier, is due the sensitivity of the ion trajectories to statistical density noise at low values of ion velocity (or temperature).

As with the distributions shown in Fig. 6, the potential (and density) is depressed at the plate by low values of ion temperature. This gives rise to higher values of electric field which accelerates ions to the plate and therefore compensates for their lack of thermal velocity.

Fig. 8 gives the velocity distributions of ions $f(u)$ at the plate for the cases where $T_{i} / T_{e}=0.1,1.0,10.0$. Again, good agreement is obtained between the exact results according to Emmert et al and those of the IMC technique. Note that the distribution function next to the plate has essentially zero particles with $u<0$, since the plate is assumed to be perfectly absorbing. These distributions clearly demonstrate the acceleration of ions towards the plate, which is strongly pronounced the lower the ion temperature.

The source function for ions used by Emmert et al was chosen to give a Maxwellian distribution in the absence of an ambipolar electric field. In the case of the high ion temperature $T_{i} / T_{e}=10.0$ in Fig. 8, the electric field is small and thus the distribution function is approximately a Maxwellian. (In this case, next to the plate, a half-Maxwellian, since no particles can leave the plate.)

The agreement between the IMC and the exact distribution function with $T_{i} / T_{e}=0.1$ is not as good as in the higher values of $T_{i} / T_{e}$. At $T_{i} / T_{e}=0.1$ the IMC case appears to broadened with respect to the exact case. This is another example of the effect of density noise on the trajectories of the ions at low values of velocity or ion temperature. The statistical noise tends to broaden the distribution.

\section{Comparison with Scheuer and Emmert}

In addition to the work of Emmert et al, Scheuer and Emmert [11] have also derived exact analytic solutions to the problem of collisionless one-dimensional plasma flow to a surface. In their case, they have used a somewhat different source velocity distribution function to that used by Emmert et al, as shown in Fig. 2. Scheuer and Emmert use a Maxwellian source function which gives rise to a thermal distribution of velocities, with a zero birth velocity being most 
probable. This contrasts with that used by Emmert et al, where zero birth velocity has zero probability. In addition, Scheuer and Emmert assume that their source strength is proportional to the local plasma density while in the case of Emmert et al a uniform source strength is used.

The choice of a different source function has a number of effects on the derived density and potential distributions (see review by Bissell et al [12]). The primary effect of having ions born with zero initial velocity is to increase the size of the electric field to accelerate the slow moving ions. Thus, the potential at the plate, and therefore the density, is depressed in the case of Scheuer and Emmert in comparison to Emmert et al. This is demonstrated in Fig. 7 which shows the potential next to the plate $-e \phi_{1} / k T_{e}$ as a function of the ratio of ion temperature to electron temperature $T_{i} / T_{e}$ for the exact solution by Scheuer and Emmert and a number of cases using their source function and the IMC technique. As with Emmert et al, good agreement is obtained for the potential.

Scheuer and Emmert have nominally solved the same problem, with the same source function, as Bissell and Johnson [10], but using a somewhat different boundary condition, i.e. Bissell and Johnson assume the Bohm criterion as a boundary condition, while Scheuer and Emmert (as well as the present work) does not. Their results differ slightly from Bissell and Johnson and they suggest this may be due to numerical inaccuracies, but may also be due to the different boundary assumption. In any case, the present analysis uses the same boundary assumptions as Scheuer and Emmert and tends to give better agreement with their results.

The source used by Scheuer and Emmert (and Bissell and Johnson) is a reasonable approximation for the case where the volume source is due to electron ionization of a hot Maxwellian gas at a temperature of $T_{i}$, while the source used by Emmert et al approximates the case where the flux tube connecting the plates is supplied by cross-field diffusion from a Maxwellian plasma. As far a simulating the boundary region of a magnetic fusion device is concerned, the source assumed by Scheuer and Emmert is a reasonable selection in the high recycling, high density regime, where significant local ionization occurs within the scrape-off layer [1]. The choice by Emmert et al is more applicable to the low recycling, low density regime, where the scrape-off layer is supplied by cross-field diffusion rather than by ionization [1].

\section{Discussion}

In this paper a new means of boundary plasm simulation has been demonstrated, the Iterative Monte Carlo (IMC) technique. In many ways, it represents a natural extension of the Monte 
Carlo (MC) technique developed by Brooks [3] and Stangeby [4], which uses a fixed background plasma. In the IMC technique, the density derived by the ion trajectories is fed back into the simulation to determine the plasma potential distribution and thus the electric field. In the results presented in this paper, the density, or cell population count, in each bin is continuously updated and thus so is the electric field. The iteration is thus essentially continuous. In other variations of this technique [13-15], applied to Langmuir probes, the iteration step is discrete, i.e. the electric field distribution is held constant while a sequence of particles modify the density distribution. The electric field is then recalculated. The process is repeated until a converged solution is obtained. In Ref. 15, of order 10 to 100 iterations is used compared with the continuous iterative scheme used here.

As a first test of the technique, a rather simple problem has been addressed, that is, collisionless one-dimensional flow to a surface. This idealized physical problem approximates the situation that exists in the scrape-off layer of magnetic fusion devices and has conveniently been solved, using two different velocity sources, using exact kinetic approaches. Good agreement between the IMC technique and the two kinetic solutions has been obtained over a wide range of the parameter $T_{i} / T_{e}$, i.e. $0.1<T_{i} / T_{e}<10$. This range encompasses most experimental and calculated estimates of the ion to electron temperature ratio in the scrape-off layers of magnetic fusion devices [1]. In addition, the two source functions represent the two extremes by which the scrape-off layer is fed with particles, i.e. in the case of Emmert et al, a cross-field diffusive source is approximated, while in the case of Scheuer and Emmert, a volume ionization source is used.

Although reasonable agreement is obtained at the lower values of ion temperature, i.e. $T_{i} / T_{e} \sim 0.1$, it should be noted that considerably more effort must be extended to achieve reliable results at these low values of ion temperature in comparison to higher values of ion temperature. This is primarily due to the fact that the statistical noise in the density distribution, and therefore the potential distribution, has a significant effect on the ion motion at low values of ion velocity. The solution to this problem is straightforward, but time-consuming; that is, simply run more particles with smaller time-steps.

It should be pointed out that the IMC technique does not address time-varying problems, such as plasma fluctuations or waves. Although statistical density "fluctuations" are present on the path to a converged solution, they have no correspondence to actual physical phenomena. 
One attraction of the IMC technique is its simplicity. The physical basis is simply two equations for particle motion -- one for the electrons and one for the ions. In the case of the electrons, the equation of motion has been still further simplified to the Boltzmann relation. Such a step is usually justified in the scrape-off layer of magnetic fusion devices. In the case of the ions, momentum must be considered and Eqns. 2 and 3 are used. In addition to these, a particle source function must be specified, which for the present comparison was quite simple, but could easily be made more complex. This situation is to be contrasted with the analytic kinetic approach, which produces, even for a simple source function, rather complex transcendental solutions.

\section{$\underline{8 \text { Conclusion }}$}

Although the problem presently addressed with the IMC technique is in fact too simple to have relevance to practical conditions present in the boundary of existing or future magnetic fusion devices, it is easy to imagine extending this technique to include a more realistic geometry and more of the relevant physical processes. For example, as with the existing impurity Monte Carlo codes [3,4], two dimensional geometry could be added, where classical plasma transport processes, i.e. collisions, dictate the parallel field ion motion and a specified "anomalous" crossfield diffusion determines the random-walk of ions across field lines. In addition, the relevant atomic processes of ionization, line radiation and recombination could be included. In short, all of the physics presently residing in existing impurity Monte Carlo codes could be included, with the added advantage that the background plasma be determined self-consistently.

\section{Acknowledgements}

This work was supported by U.S. Department of Energy Contract No. DE-AC02-76-CHO3073 and No. DE-AC04-76DP00789. C S Pitcher is grateful for personal support from the Canadian Fusion Fuels Technology Project and to P C Stangeby for suggesting the comparison with the work of Emme:t et al. 


\section{References}

(1) P C Stangeby and G M McCracken, Nucl Fus 30 (1990) 1225.

(2) D L Hillis, J T Hogan, M R Wade et al, "Helium Transport Studies in Enhanced Confinement Regimes on the DIII-D Tokamak", presented at the 14th Int Conf on Plas Phys and Contr Nucl Fusion Research, Wurzburg, Germany, 30 Sept - 7 Oct 1992, IAEA CN-56/A-7-20.

(3) J N Brooks, J Nucl Mat 145-147 (1987) 837.

(4) P C Stangeby, C Farrell, S Hoskins and L Wood, Nucl Fus 28 (1988) 1945.

(5) G F Matthews, J D Elder, N A C Gottardi et al, J Nucl Mat (1992), to be published, presented at the 10th Int Conf PSI in Contr Fus Devices, Monterey, March 1992.

(6) G M McCracken, P C Stangeby, D H J Goodall et al, Fus Eng Des 11 (1989) 370.

(7) P C Stangeby, The plasma sheath, in "Physics of Plasma-Wall Interactions in Controlled Fusion", Eds. D E Post and R Behrisch, NATO ASI Series, Plenum Press, New York (1986) 41 .

(8) L Tonks and I Langmuir, Phys Rev 34 (1929) 876.

(9) G A Emmert, R M Wieland, A T Mense and J N Davidson, Phys Fluids 23 (1980) 803.

(10) R C Bissell and P C Johnson, Phys Fluids 30 (1987) 779.

(11) J T Scheuer and G A Emmert, Phys Fluids 31 (1988) 3645.

(12) R C Bissell, P C Johnson and P C Stangeby, Phys Fluids B1 (1989) 1133.

(13) L W Parker and E C Wipple, Annals of Physics 44 (1967)126.

(14) Å Skøelv, R J Armstrong and J Trulsen, Phys Fluids 27 (1984) 2744.

(15) R J Armstrong, K -B Liland and J Trulsen, Physica D (1992) 


\section{Figure Captions}

Fig. 1 Schematic diagram of the one-dimensional problem, showing the qualitative trends of the plasma density $\mathrm{n}$ and the potential $\phi$.

Fig. 2 The source function $S(u)$ used by Emmert et al [9] and Scheuer and Emmert [11] for ions born in the space between plates is a function of normalized ion velocity $u \equiv v\left[2 k T_{i} / m\right]^{-1 / 2}$.

Fig. 3 The plasma grid used in the IMC technique. Note the bin separation $h$ is not necessarily constant.

Fig. 4 Results during an IMC simulation (after approximately 200 particles with $T_{i} / T_{e}=0.1$ ) showing (1) the normalized density proile $n(s) / n_{0}$ just before and just after a particle whose trajectory is strongly affected by density "noise" (2) the trajectory of this particle, showing it originally trapped in a potential well and then escaping as the well is filled in with "density". The right vertical axis shows the numbur of trajectory time-steps.

Fig. 5 History traces for the normalized density at the plate $n(L) / n_{0}$ for two separate simulations with $T_{i} / T_{e}=1$ : (1) the Iterative Monte Carlo (IMC) technique, where the cell population count is fed back to derive the electric field (2) the standari Monte Carlo (MC) technique, where the exact electric field according to Emmert et al [9] is used throughout the simulation. Also shown is the exact solution for the densit at the plate according to Emmert et al, $n(L) / n_{0}=0.667$.

Fig. 6 The normalized density $n(s) / n_{0}$ as a function of normalized position $s \equiv x / L$ for three values of $T_{i} / T_{e}=0.1,1,10$. Comparison between the exact solution by Emmert et al and results using the IMC technique.

Fig. 7 The normalized potential next to the plate $-e \phi_{1} / k T_{e}$ as a function of the ratio of ion temperature to electron temperature $T_{i} / T_{e}$ for the exact solution by Emmert et al [9] and Scheuer and Em*1ert [11] and for a number of corresponding cases using the IMC chnique. 
Fig. 8 The velocity distributions of ions $f(u)$ at the plate for the cases where $T_{i} / T_{e}=0.1,1,10$. Comparison between the exact solution by Emmert et al and results using the IMC technique. 


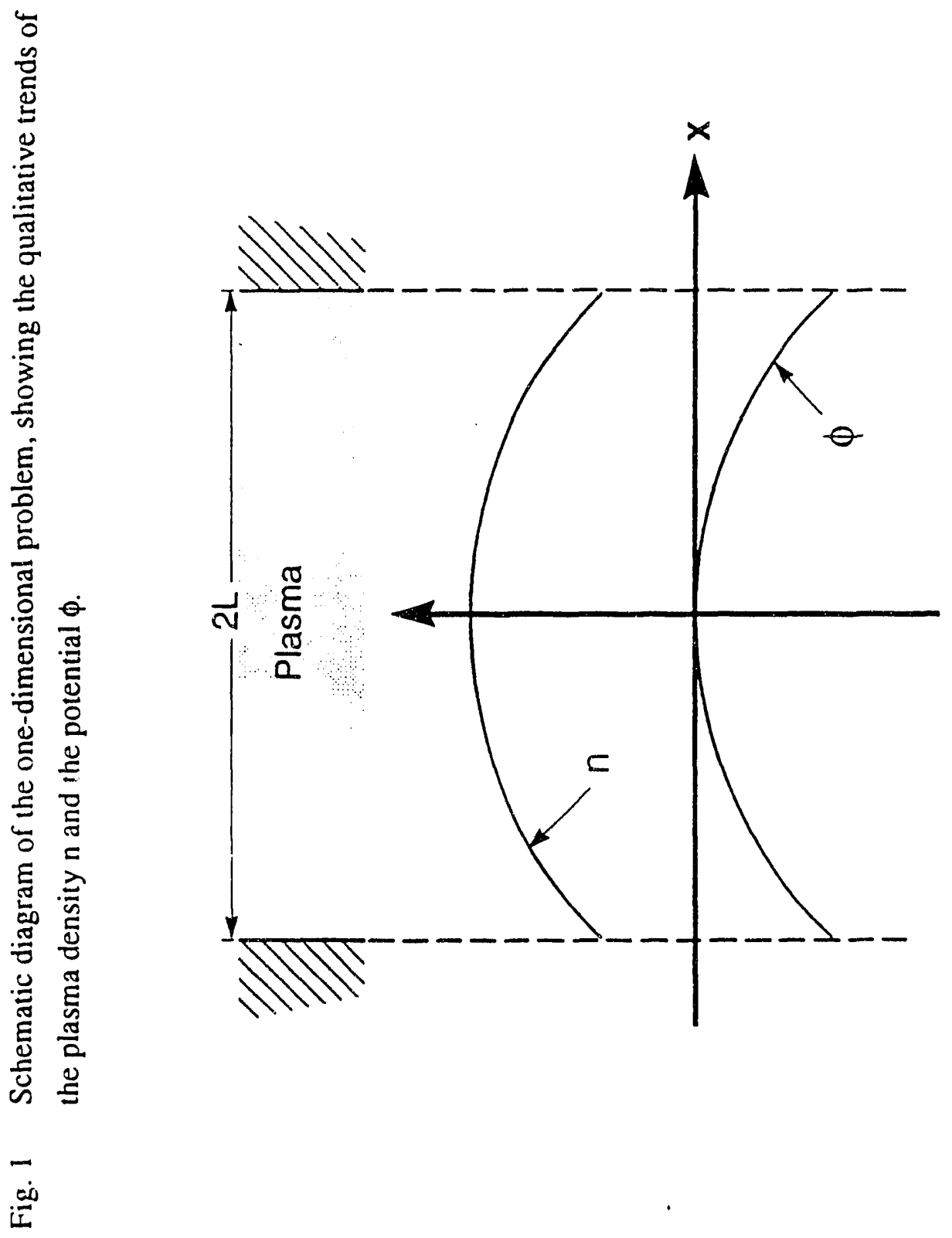




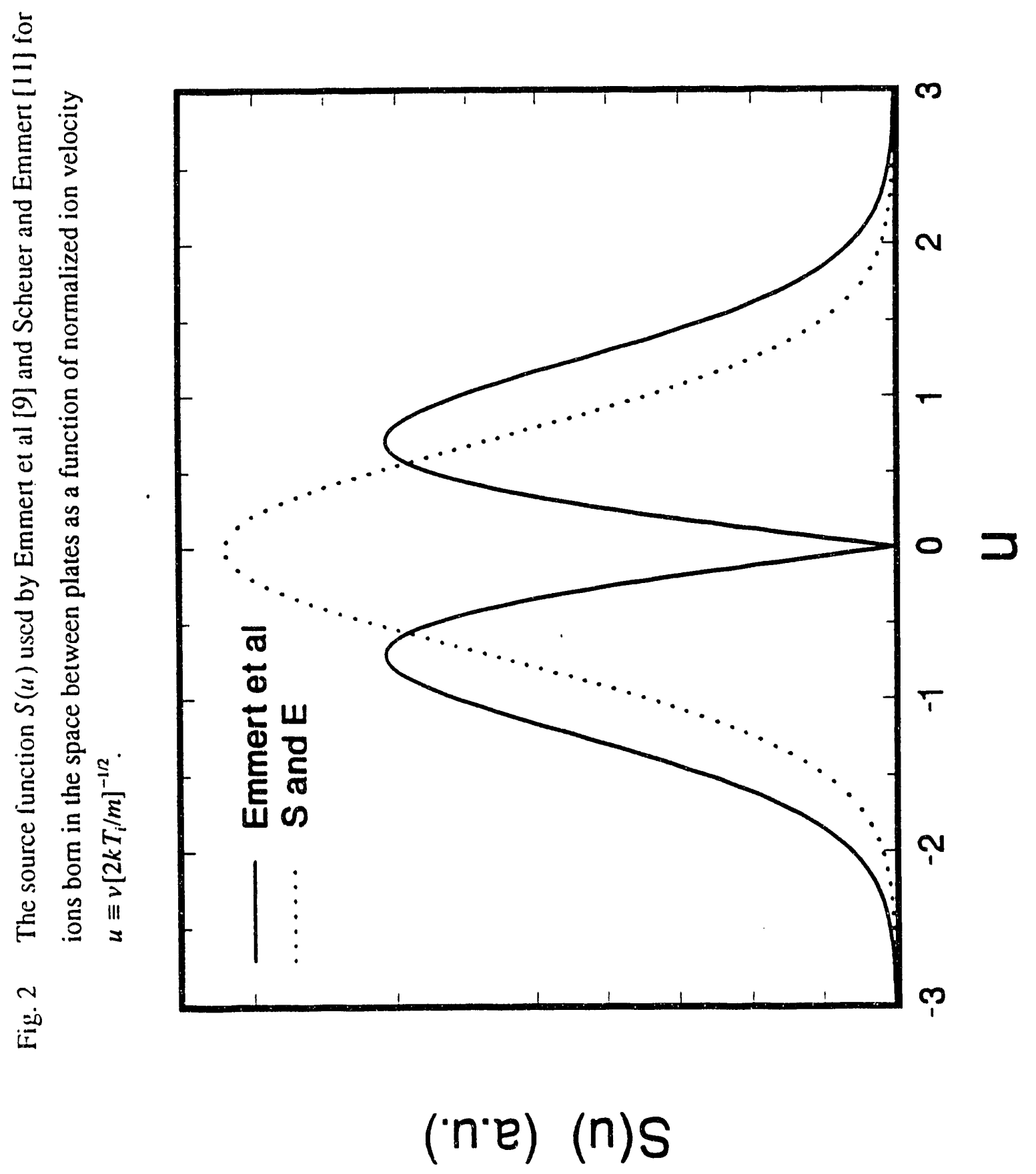



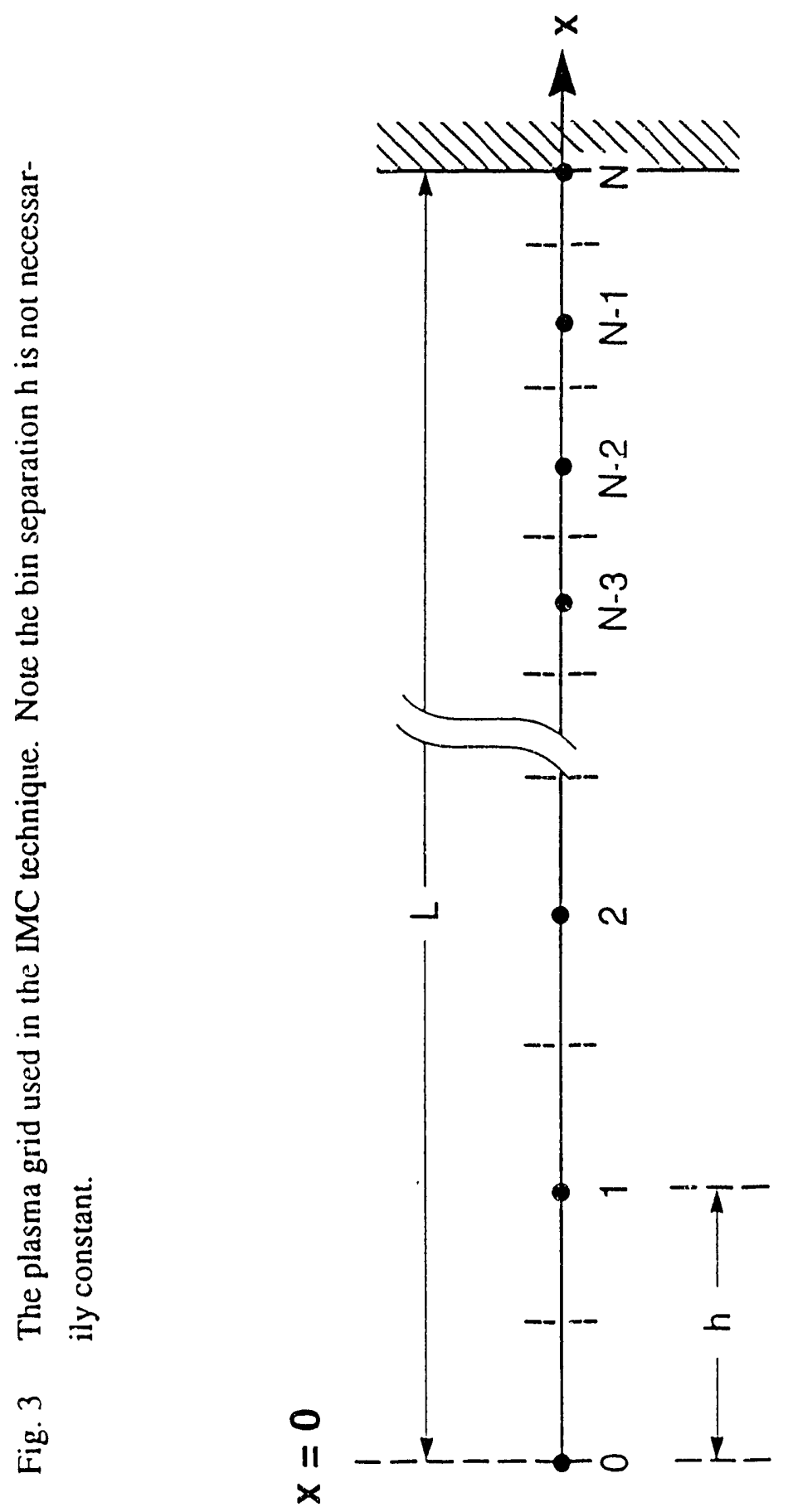


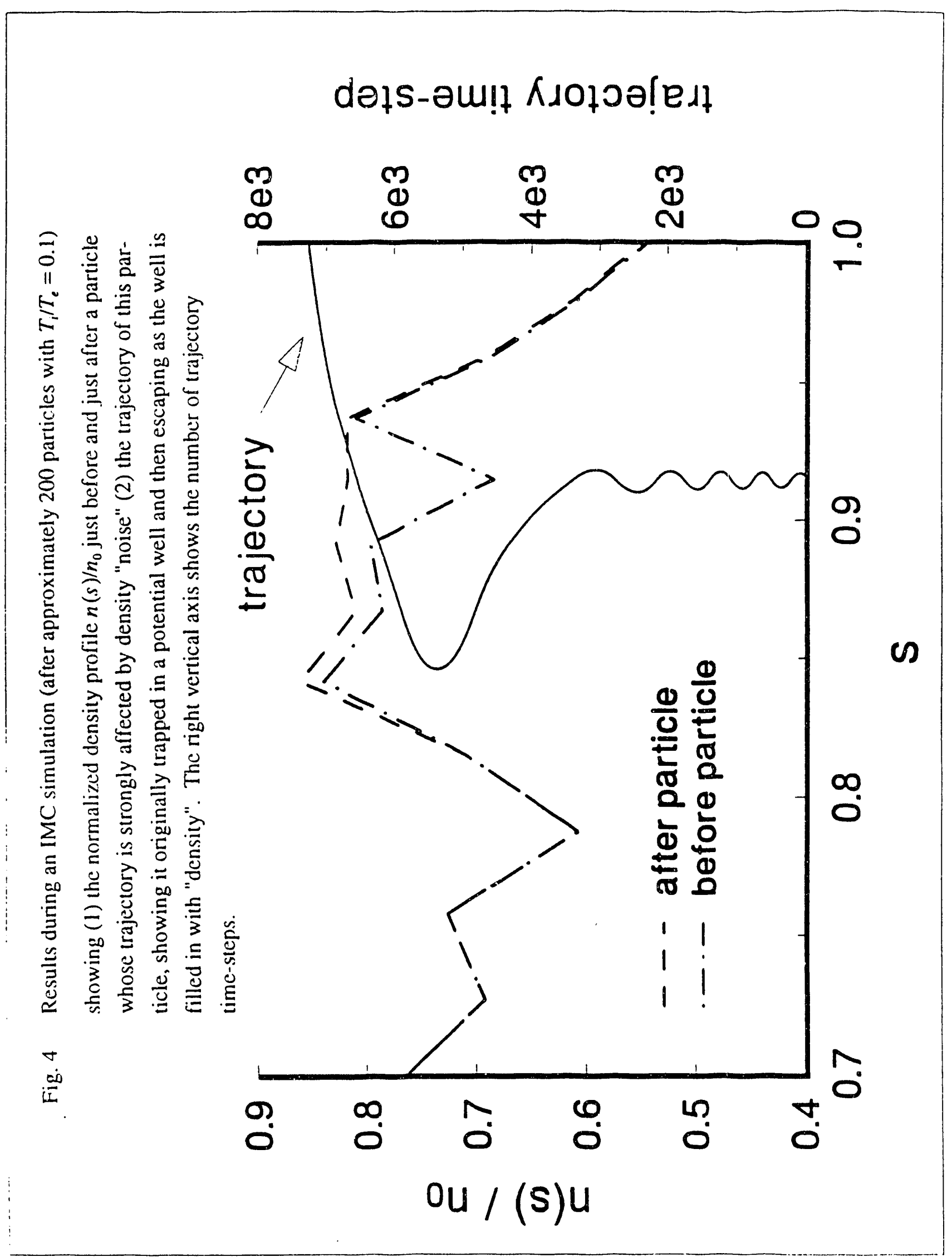




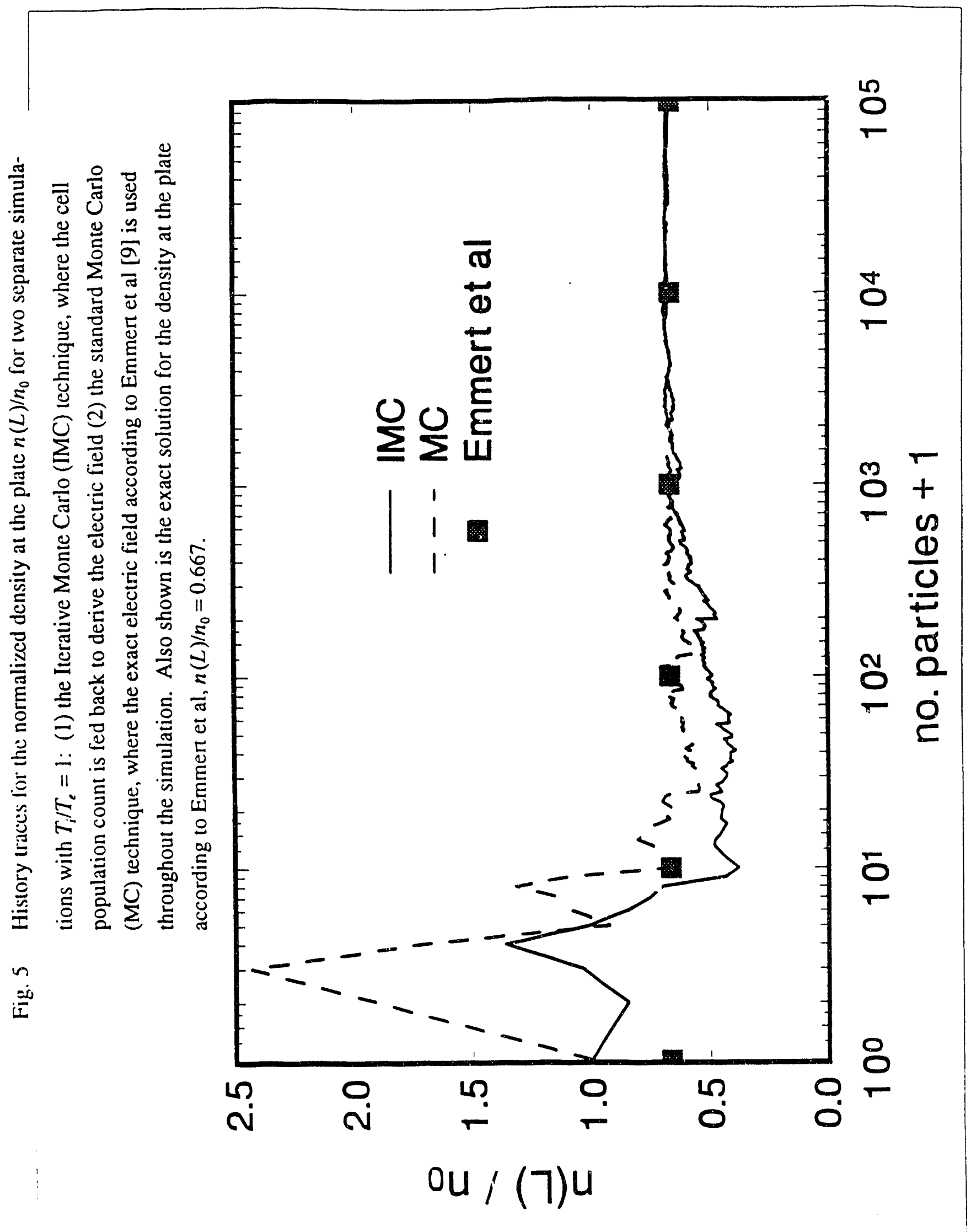




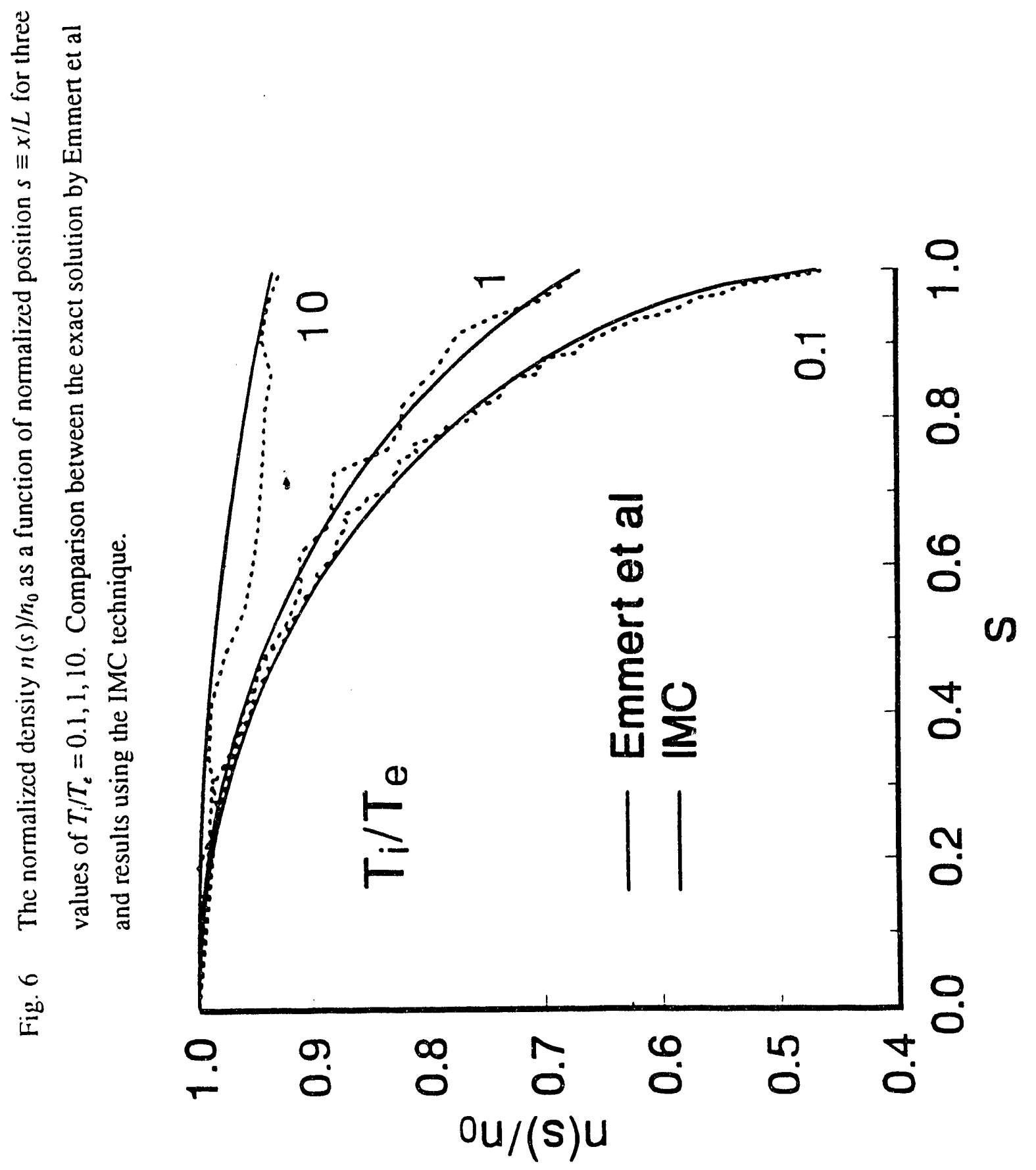




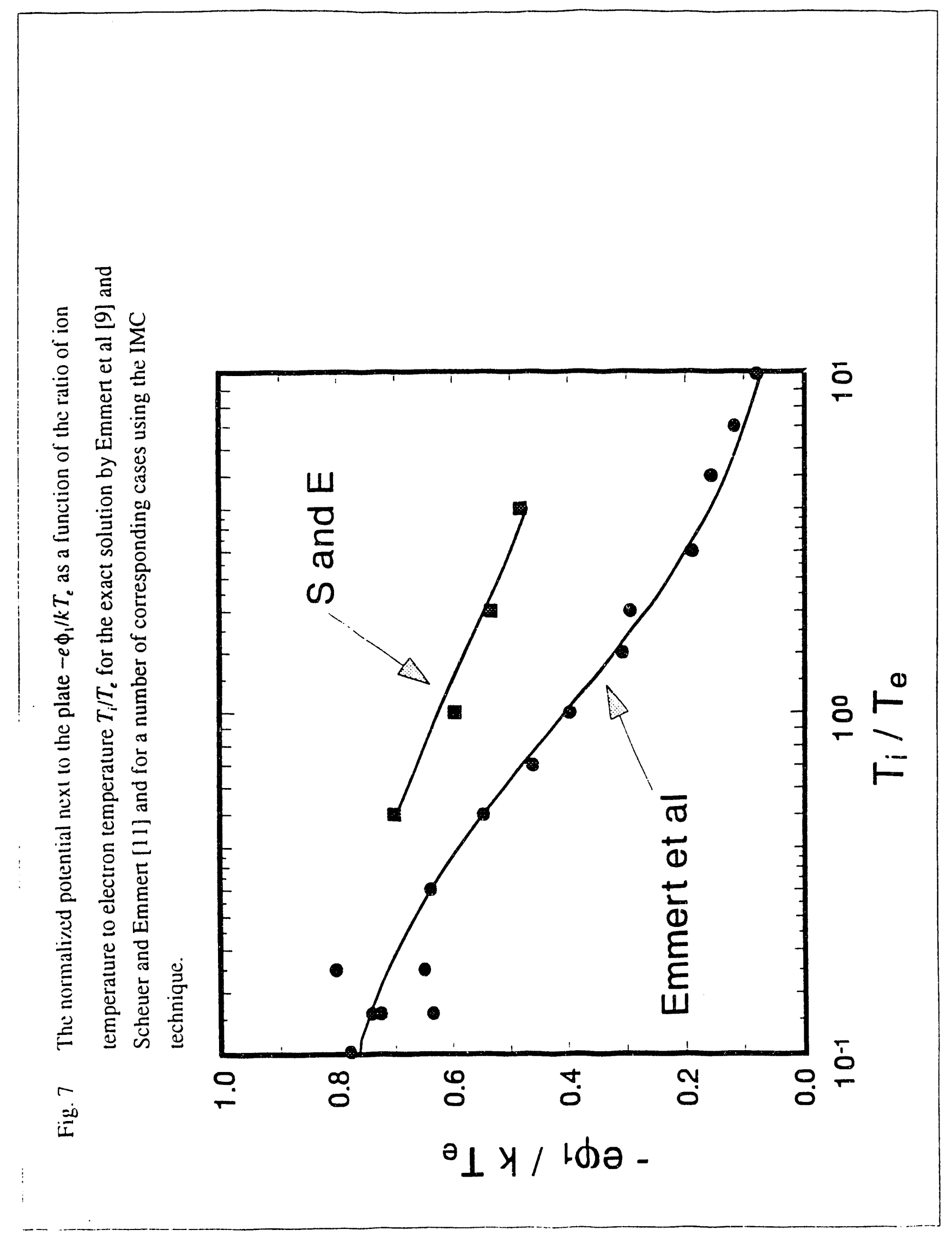




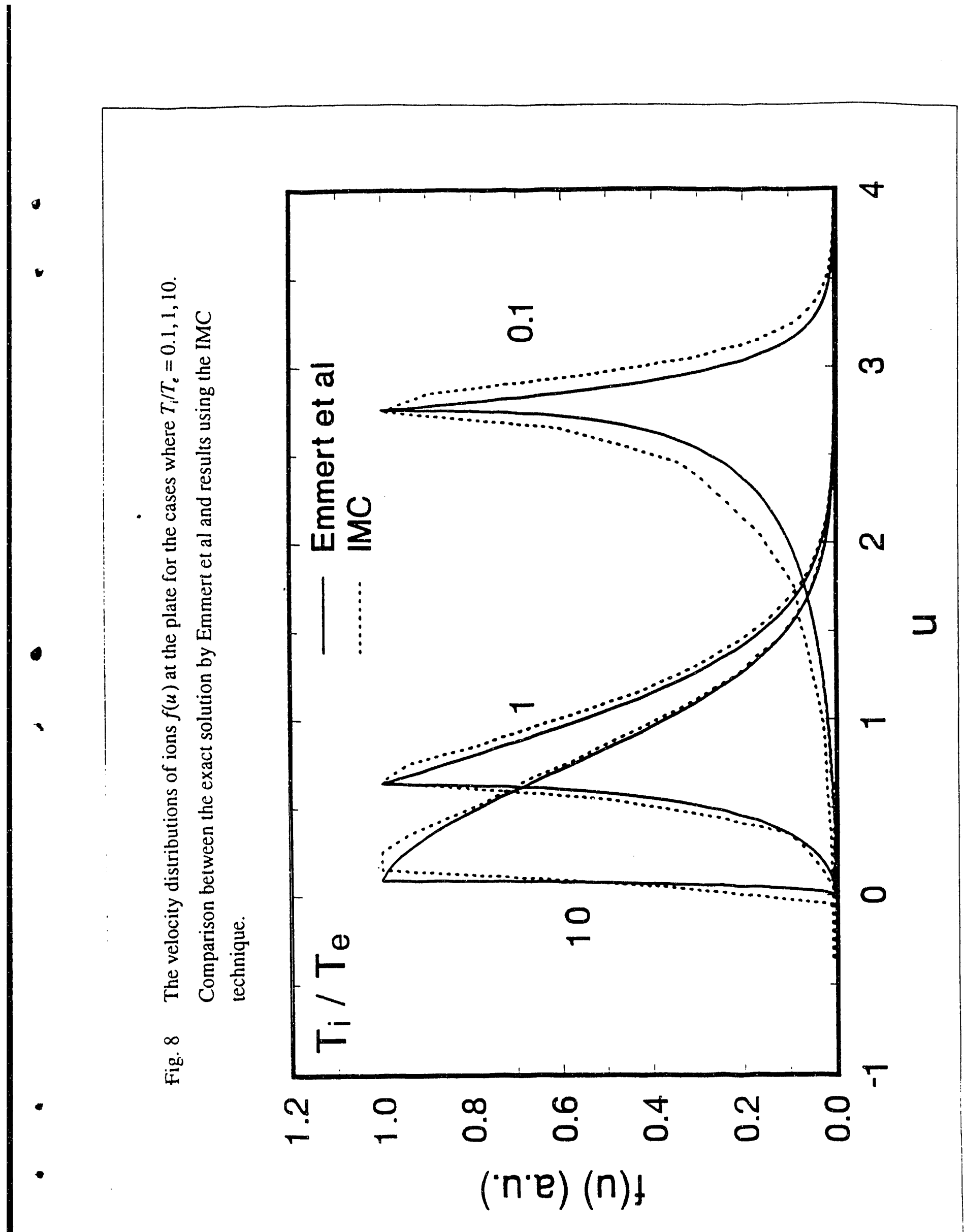


Dr. F. Peotoni, Univ. of Wollongong. AUSTRALIA
Prof. M.H. Brennan, Univ. of Sydney, AUSTRALIA

Plasma Rosearch Leb., Austrelien Nal. Univ., AUSTRALIA

Prof. I.R. Jones, Flindors Univ, AUSTRALIA

Prot. F. Cap, Inst for Theoretical Physics, AUSTRIA

Prol. M. Heindier, Instiut for Theoretieche Phyeik, AUSTRIA

Prot. M. Gooscons, Astronomisch InetituUt, BELGIUM

Ecoto Royed Mlitairs, Leb. de Phy. Plasmas, BELGIUM

Cornmission-Europen, DG. XII-Fusion Prog., BELGIUM

Prof. R. Bouciqus, Rijkeuniveribit Gent, BELGIUM

Dr. P.H. Sakenake, Instituto Fivica, BPLZIL

Instituto Necioned Do Peaquiase Espaciaiz-INPE, BPUZIL

Documents Olifos. Abmic Energy of Ceneda Lid., CANADA

Dr. M.P. Bectyneki, MPB Tectnologios, Inc., CANADA

Dr. H.M. Skereoard, Univ. of Sackatchowen, CANADA

Prof. J. Teichmam, Univ. of Montred, CANADA

Prot. S.R. Srecrivecen, Univ. of Celoany. CANADA

Prof. T.W. Johneton, INAS-Energio, CANADA

Dr. R. Bolion, Contre canedien de tusion magnetique, CANADA

Dr. C.R. Jamee., Univ. of Aberta, CANAOA

Dr. P. Lukte, Komonatetho Univerezita, CZECHOSLOVAKIA

The Librerien, Cuham Leboratory. ENGLAND

Librey, R61, Ruthertord Appleton Laboratory, ENGLAND

Mrs. S.A. Hutchinsoon, JET Librery, ENGLAND

Dr. S.C. Sheme, Univ. of South Pacific, FIJI ISLANDS

P. Menonen, Univ. of Holeinki, FINLAND

Prof. M.N. Busenc, Ecolo Potyluctinique., FPANCE

C. Mourter, Lob. do Physique des Milioux loniseds, FRANCE

J. Radat, CENCADARACHE - Bat 506, FRANCE

Prof. E. Economou, Univ. of Crow, GREECE

Ma. C. Rinni, Unir, of lommina, GREEGE

Dr. T. Muel, Acadermy Bbilogrephic Ser., HONG KONG

Proprint Librery. Hungarian Acadorny of Sa.. HUNGARY

Dr. B. DesGupte, Saha Inat of Nuctioer Phyeica, INDIA

Dr. P. Kew, Inct. for Pleema Recearch, INDLA

Dr. P. Poseneu, Iereet inat of Technology, ISPAEL

Librarien, Intemaciond Conter for Theo Physics, ITALY

Miss C. De Palo, Associazions EUAATOMENEA, ITALY

Dr. G. Groseo, leviuto d Ficica ded Plasma, ITALY

Prol. G. Postengni, latuto Ges lonizzai Del Cnr, ITALY

Dr. H. Yanneo, Toehibe Res a Dowel Conter, JAPAN
Prof. I. Kewakami, Hiroshima Univ., JAPAN

Prof. K. Nishikewa, Hiroshima Univ., JAPAN

Director, Jepen Atomic Enorgy Rosearch Inst, JAPAN

Prof. S. Ioh, Kyushu Univ., JAPAN

Roserect into. Cr., National Instil for Fusion Science, JAPAN

Prof. S. Tenaka, Kyoto Univ., JAPAN

Librery. Kyoto Univ., JAPAN

Prot. N. Inow, Univ. of Tokyo, LAPAN

Secretary, Pleame Section, Elbatrownical Lab., JAPAN

S. Mori, Techniced Advieor, LAERI, LAPAN

Dr. O. Nuinri, Kumanoso Inst of Tectnobogy. JAPAN

J. Hproon-Sook, Korea Abomic Energy Rasearch Inst, KOREA

D.I. Chai, Tho Korea Adv. Inst of Sai. \& Tech., KOREA

Prot. B.S. Liby, Univ. of Weikat, NEW ZEALAND

Inat of Phyrias, Chines Acad SA PEOPLE'S REP. OF CHINA

Librey, Inst of Plasma Physics, PEOPLE'S REP. OF CHINA

Teinginu Univ. Libray. PEOPLE'S REPUBLIC OF CHINA

2. Li, S.W. Ina Phyeica, PEOPLE'S REPUBUC OF CHINA

Prof. J.A.C. Gebred, Instiuto Superior Tecnioo, PORTUGAL

Dr. O. Potrus, ALI CUzA Univ., ROMANIA

Dr. J. do Viliors, Fusion Studios, AEC, S. AFRICA

Prof. M.A. Hemborg, Univ. of Natid, S. AFRICA

Prof. D.E. Km, Poheng Inte. of Sci. \& Tech., SO. KOREA

Prof. C.I.E.M.A.T, Fusion Division Library. SPAIN

Dr. L Simnio, Univ. of UAEA, SWEDEN

Librey, Royd inet of Technology, SWEDEN

Prof. H. Whalmeon, Chalmore Univ, of Tech., SWEDEN

Cente Phys. Des Plasmas, Ecolo Potyoch, SWITZERLAND

Bibliotheck, Inat. Voor Phema-Fyeice, THE NETHERLANDS

Aset Prot. Dr. S. Cakir, Middo East Tech. Univ., TURKEY

Dr. V.A. Gulhikh,Sd. Res. Inst Electrophys.I Appearatus, USSR

Dr. D.D. Pyutov, Siberian Brench of Academy of Sai., USSA

Dr. C.A. Eliceov, I.V. Kurchator Inat, USSA

Librarien, The Uwr.SSA Acodomy of Scionces, USSR

Dr. L.M. Kovriathnykh, Inst. of Generd Physics, USSA

Kemtonchungeanlege GmbH, Zentrabibliothek, W. GERMANY

Bibliothate, Inse For Phamatorechung, W. GERMANY

Prof. K. Schindier, Ruhr-Universitu Bochum, W. GERMANY

Dr. F. Weoner, (ASOEX), Max-Planck-Institut, W. GERMANY

Librerien, Max-Plenck-Institut, W. GERMANY

Prof. R.K. Jenov, Inet of Phycics, rucosLavia 

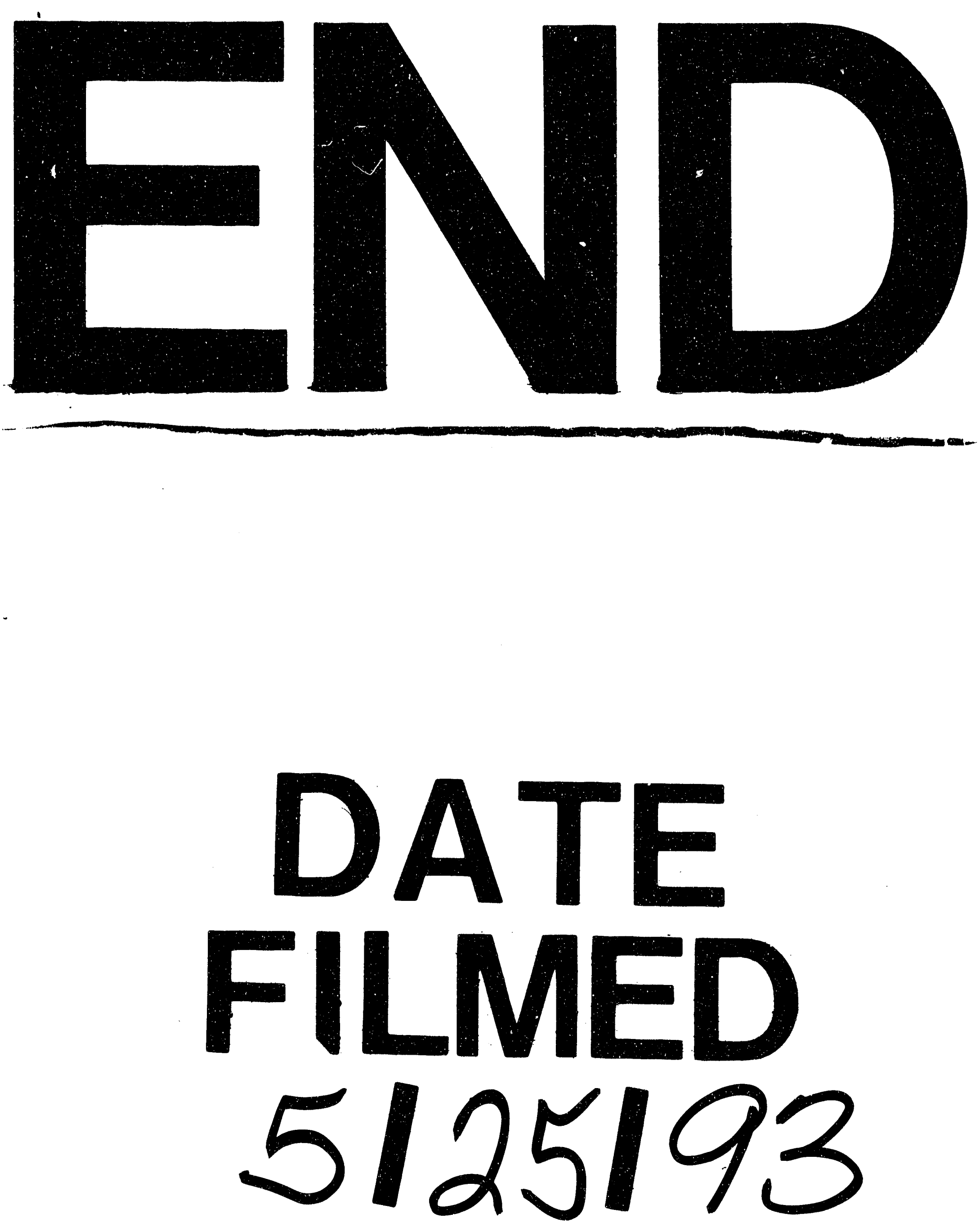

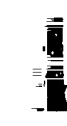


